\title{
Carbon Nanotubes in Neuroscience
}

\author{
Erik B. Malarkey and Vladimir Parpura \\ Department of Neurobiology Center for Glial Biology in Medicine, Atomic Force Microscopy and \\ Nanotechnology Laboratories, Civitan International Research Center, Evelyn F. McKnight Brain \\ Institute, University of Alabama 17196th Avenue South, CIRC 429, Birmingham AL 35294, USA \\ Vladimir Parpura: vlad@uab.edu
}

\begin{abstract}
Carbon nanotubes have electrical, mechanical and chemical properties that make them one of the most promising materials for applications in neuroscience. Single-walled and multi-walled carbon nanotubes have been increasingly used as scaffolds for neuronal growth and more recently for neural stem cell growth and differentiation. They are also used in interfaces with neurons, where they can detect neuronal electrical activity and also deliver electrical stimulation to these cells. The emerging picture is that carbon nanotubes do not have obvious adverse effects on mammalian health. Thus in the near future they could be used in brain-machine interfaces.
\end{abstract}

\section{Keywords}

Carbon nanotubes; modification; substrates/scaffolds; electrical interface; neurobiology

\section{Introduction}

The first evidence of nano-sized carbon tubes (Fig. 1a) is thought to have been shown by Radushkevich and Lukyanovich in 1952 (1). Several other groups observed similar carbon structures afterwards but it was the efforts of two groups in $1993(2,3)$ that stirred the current interest in carbon nanotubes (reviewed in (4)).

Carbon nanotubes (CNTs) are composed of sheets of graphene formed into a cylinder, either a single cylinder, single-walled carbon nanotube (SWNT) or multiple concentric cylinders, multi-walled carbon nanotube (MWNT). Double-walled CNTs (DWNTs) are composed of two concentric graphene cylinders, and they represent an intermediate structure between MWNTs and SWNTs. The size of CNTs typically ranges from 0.4 to $2 \mathrm{~nm}$ in diameter for SWNTs and 2 to $100 \mathrm{~nm}$ for MWNTs while their length can vary from one to several hundred micrometers. The arrangement of the carbon atoms in the graphene sheet can take on several conformations, armchair, chiral or zigzag. These conformations of the nanotube determine its conductivity. Nanotubes are most commonly synthesized upon a catalyst by a variety of methods including, chemical vapor deposition, electric arc discharge and laser ablation.

After manufacture, CNTs are often modified to improve their biocompatibility or enable them to perform new functions by attaching various compounds to them. Lipids, DNA and various peptides can often be simply adsorbed to the CNT. If a more permanent attachment

Correspondence to: Vladimir Parpura, vlad@uab . edu.

Conflict of interest statement We declare that we have no conflict of interest. 
is desired, compounds may be covalently linked to nanotubes. This is most often done by incubating nanotubes with strong oxidizing agents like nitric acid, which add carboxyl groups to the ends of the tubes and any defect sites. Other groups can then be added, usually converting the carboxyl group to acyl chloride, which can then be reacted with the compound of interest. For review on CNT structure and modifications see $(5,6)$.

In this review we discuss the use of CNTs in neuroscience, focusing mainly on recent developments with regard to their use as scaffolds for neuronal growth and as electrical interfaces, with brief coverage of earlier studies. For review on the earlier applications of CNTs in neurobiology see (7).

\section{Carbon Nanotubes as Substrates/Scaffolds for Neural Cell Growth}

CNTs have been studied as substrates for neuronal growth. Their size and shape are similar to neuronal processes; they are strong yet flexible and can be made conductive. These are all qualities that are advantageous for creating scaffolds for neuronal growth. The first demonstration that nanotubes could be used as a substrate for neurons was provided by Mattson et al. (8) who grew hippocampal neurons on glass coverslips coated with the permissive substrate polyethyleneimine (PEI) and overcoated with MWNTs. They also discovered that they could improve the growth of neurons by modifying the MWNT with a biologically active compound, 4-hydroxynonenal; this modification was simply done by physiosorption. This study was followed up by Hu et al. (9) who grew neurons on MWNT substrates with different charges to investigate whether nanotube substrates could be systematically modified in order to affect neurite outgrowth and branching. By imaging using the vital dye, calcein, they found that when compared to the commonly used substrate, PEI, neurons on as-prepared MWNT substrate (Fig. 1b) did not grow as well, although MWNTs did act as a permissive substrate. They next modified the charge of the MWNT substrate by functionalizing it with carboxyl groups, poly-m-aminobenzene sulfonic acid or ethylenediamine to create negatively, zwitterionic or positively charged nanotubes, respectively. They found that as the substrate became more positive the length and branching of neurites increased. In a subsequent study Hu et al. (10) found that culturing neurons on substrates made of a graft copolymer, where SWNTs were functionalized with branched PEI to "dilute" PEI's positive charge, resulted in neurite outgrowth and branching intermediate to that of as-prepared MWNTs or PEI alone. Some modifications have been shown to decrease the bio-compatibility of nanotubes. Liopo et al. (11) showed that 4tertbutylphenyl or 4-benzoic acid functionalized SWNTs were less supportive of neural cell attachment and growth than unfunctionalized nanotubes.

Covalent modifications of CNTs, which increase the retention of an attached compound/ group, can also be achieved with proteins that play a role in brain signaling. For example, neurotrophins are protein growth factors that promote the survival and differentiation of neurons. Two such proteins, nerve growth factor (NGF) and brain-derived neurotrophic factor (BDNF), covalently bound to MWNTs, could be used to regulate the growth of neurons (12). Adding the MWNTs coated with NGF or BDNF to the culture medium of embryonic chick dorsal root ganglion cultures promoted neurite outgrowth similar to soluble NGF or BDNF. These results indicate that biologically active molecules like neurotrophin bound to CNTs can retain their activity and interact with cells to promote their function.

Beyond depositing nanotubes onto planar glass coverslips, freestanding films of a poly $(\mathrm{N}-$ cetyl-4-vinylpyridinium bromide-co-N-ethyl-4-vinylpyridinium bromide-co-4-vinylpyridine) SWNT copolymer have been created and used as a substrate for neural cell growth (13). Furthermore, Galvan-Garcia et al. (14) reported that directionally oriented MWNTs in the form of sheets or yarns offer an alternative presentation of CNTs to cells. These CNT forms promoted cell attachment, differentiation and cell growth. Additionally, 
when highly purified, these CNTs also allowed neurons to extend processes of which the number and length were comparable to those of neurons grown on a planar permissive substrate, represented by polyornithine pre-treated glass. Thus, the interaction between neurons and CNTs may be affected by the purity of CNTs as well as by the 3-dimensional organization of the CNT substrate/scaffold. Interestingly, using a particle coagulation spinning process, carbon threads and ribbons up to $30 \mathrm{~cm}$ in length can be made from SWNTs (15). These nanomaterials were also compatible with neural cells as demonstrated by culturing hippocampal neurons and PC12 cells on them. After a week in culture, neurite outgrowth on the nanothread surface could be observed by immunolabeling against $\beta$ IIItubulin, a neuron specific label. Soluble NGF that can induce neurite outgrowth from PC12 cells was able to exert such an action on PC12 cells grown on CNT threads. This demonstration of the biocompatibility of these nanothreads indicates that they may be suitable for the construction of electrodes or nanowires in implantable devices.

Gabay et al. (16) created a micropatterned array of CNT islands to study self-organization of neural networks. They used a poly (dimethyl siloxane) stamp to imprint a pattern of iron nanoparticle islands on quartz substrates and used chemical vapor deposition to grow CNTs on the iron catalyst islands. The neurons they cultured on these substrates had accumulated on the CNT islands within 4 days with neuronal processes seen to bridge across the nonpermissive quartz to form connections between adjacent islands. Similarly, Sorkin et al. (17) created a SWNT patterned substrate by applying iron nitrate catalyst to coverslips with a polydimethylsiloxane stencil and then growing CNTs by chemical vapor deposition. Hippocampal neurons were cultured on these substrates and after 2 to 3 days spontaneously grew into islands with neurites connecting each island. These patterned networks could be used to study neuronal networking with CNT islands as electrical connections for sensing or stimulation.

Besides the above outlined studies on neurons, there have been some attempts to use CNTs in stem cell research. The growth and differentiation of neural stem cells on nanotube substrates was recently demonstrated (18). Coverslips coated with six layers of SWNTs dispersed in poly(sodium 4-styrene-sulfonate) and PEI were used to culture neural stem cells, along with coverslips coated with only poly-L-ornithine for comparison. Cells were bathed in epidermal growth factor (EGF)-depleted medium to induce differentiation. Cells adhered and differentiated into neurons, astrocytes and oligodendrocytes as shown by immunoreactivity of nestin, microtubule-associated protein 2 (anti-MAP2), glial fibrillary acidic protein (anti-GFAP), and oligodendrocyte marker O4 (anti-O4). These cells were just as viable on the CNT substrate as on poly-ornithine and differentiated and extended processes similarly. Since there was no adverse effect of growing on nanotube substrates these materials could readily be used for applications involving stem cells.

\section{Carbon Nanotubes as Electrical Interfaces with Neurons}

One of the most exciting developments at the crossroads of nanotechnology and neuroscience is that of designing neural interfaces. Such interfaces between CNTs and neurons have been demonstrated, indeed. Wang et al. (19) patterned a microchip on a quartz substrate using plasma etching and photolithography. Iron pads were created in silicon dioxide and MWNTs were grown on them by chemical vapor deposition. The nanotubes were decorated with polyethylene glycol to make them more hydrophilic. Hippocampal neurons were cultured onto the microchips and allowed to grow for 4 days. Incubating the cells with fluo-4 allowed them to monitor $\mathrm{Ca}^{2+}$ activity during stimulation. Electrical stimulation of the neurons through the CNT microelectrodes on the chip caused rapid changes in fluo-4 fluorescence, indicating transient increases in intracellular $\mathrm{Ca}^{2+}$ concentration most likely due to $\mathrm{Ca}^{2+}$ influx after the stimulated neurons discharged action potentials. These changes in fluorescence were also seen upon application of glutamate. This 
demonstrates the feasibility of creating microchip arrays for studying neuronal networks. Liopo et al. (11) also used the conductive nature of CNT substrates to stimulate neurons cultured on them. They deposited SWNTs onto polyethylene terephthalate films and glued a ring in the middle of the film to contain dorsal root ganglion neurons. Electrodes attached to the CNT substrate outside of the ring were used to apply current through the nanotube substrate which resulted in an inward current in the neurons that resembled currents induced by depolarizing voltage steps.

Neuron-neuron signaling is mainly achieved by synaptic transmission. Initially, Lovat el al. (20) examined the effects that CNTs may exert on neuronal electrical activity. They cultured rat, postnatal, hippocampal cells on either uncoated glass coverslips or coverslips coated with unfunctionalized MWNT films. After 8 days of culturing, the neurons were probed for their electrical activity using single cell patch clamp recording. Neurons grown on MWNT films showed a sixfold greater frequency of spontaneous postsynaptic currents and spontaneous action potential generation when compared to those grown on plain glass. The authors proposed that the high conductivity of the CNT substrate might have affected voltage-dependent membrane processes resulting in the increased activity. If this is the case, then growing neurons on CNTs could be used as a platform for stimulation of neurons via CNTs to induce signaling through synaptic contacts. This was subsequently addressed by Mazzatenta et al. (21) who grew hippocampal neuronal cultures on glass coverslips coated with SWNT films. Scanning electron microscopy revealed that neurons grew on the nanotubes similarly to control glass surfaces; it also revealed tight interactions between the neuronal membranes and the SWNTs. Electrophysiological recordings indicated that neurons grown on SWNT substrates displayed spontaneous electrical activity. By applying current through the nanotube substrate they were able to induce action potentials in the cultured neurons. Also, after stimulation they could observe postsynaptic currents as an indication of neuron-neuron signaling. Taken together, the above studies show that CNTs may provide a means for electrical coupling between devices and neurons.

During synaptic transmission at pre-synaptic terminals the vesicles fuse to the plasma membrane and release their cargo molecules in response to depolarization. Besides classical transmitters, such as dopamine, additional compounds such as chromogranin A ( $\mathrm{CgA}), \mathrm{a}$ protein located in dense core vesicles/granules, can be released. We briefly outline two studies that used CNTs to improve detection of dopamine and CgA.

Ali et al. (22) generated a non-oxidative method to detect dopamine, which can be otherwise detected oxidatively, albeit such detection has several disadvantages (see details in (22)). Briefly, they found that they could increase the sensitivity of electrochemical electrodes used in the detection of dopamine by coating them with conjugated SWNTs. The SWNTs were conjugated with poly(anilineboronic acid) (PABA) which acts as a selective dopamine reactant. The binding of dopamine forms a dopamineboronate ester complex which alters the conductivity of the polyaniline backbone without oxidizing dopamine itself. They used single stranded DNA to disperse SWNTs $(23,24)$ and then attached PABA to them using electropolymerization. This composite was then applied to the electrodes. They also eliminated interference from ascorbic acid, the most common compound to interfere with the electrochemical detection of dopamine, without a loss in electrode sensitivity. This was accomplished by coating the composite with a thin layer of perfluorosulfonated ionexchange polymer, Nafion. The large surface area of the nanotubes greatly increased the amount of borate groups that could detect dopamine. They were able to detect dopamine concentrations as low as $1 \mathrm{nM}$ using cyclical voltametry and $40 \mathrm{pM}$ using differential pulse voltametry. This was $1000 \times$ more sensitive than using only PABA coated electrodes. 
Wang et al. (25) developed a SWNT based field-effect transistor (FET) to detect the release of CgA from cultured cortical neurons. The FET was created by photolithography and then coated with SWNTs. After baking, the nanotubes became connected to leads on the FET. A goat immunoglobulin $\mathrm{G}(\operatorname{IgG})$ antibody against $\mathrm{CgA}$ was immobilized on the SWNTs to enable detection of $\mathrm{CgA}$. Upon application of $\mathrm{CgA}$ an increase in current in the FET could be detected. This FET could detect $\mathrm{CgA}$ at concentrations less than $1 \mathrm{nM}$. A coverslip containing cultured neurons was placed on the FET and the cells were stimulated by the application of glutamate. This stimulation caused the release of $\mathrm{CgA}$ as detected by an increase in current in the FET. This use of nanotubes in a FET provides another method for the real time detection of molecules released from neurons and presents a possible device for sensing neural activity.

Electrodes that have been used for brain implants are metal-based, have relatively high impedance and poor charge delivery. Carbon nanotubes have several properties which could be employed to improve the performance of such microelectrodes; they have high mechanical strength to penetrate the tissue, and their ability to perform as ballistic conductors, aids in lowering the impedance and increasing the charge transfer compared to regular metal electrodes. Keefer et al. (26) successfully developed a deposition procedure for applying MWNTs to metal electrodes. They coated commercially available tungsten and stainless steel sharp electrodes with CNTs, which when tested in solution outperformed the bare metal electrodes by offering lower impedance and higher charge transfer. Two different animal models were used to test CNT-coated sharp electrodes: motor cortex of anesthetized rats and visual (V4) cortex of a trained monkey. In both in vivo experimental models, they used two parallel electrodes, one coated with CNTs and the other bare metal. CNT-coated electrodes outperformed their paired control electrodes in terms of reduced noise and increased sensitivity in the detection of spontaneous electrical neuronal activity. As one would predict from their mechanical strength, CNTs endured the advancement of electrodes through the dura mater. These hybrid electrodes could be now readily tested in the field of brain-machine interfaces (27).

\section{Concluding Remarks}

The intent of this review was to briefly discuss the use of CNTs in neuroscience, most notably as scaffolds for neuronal growth and as electrical interfaces with neurons.

It is apparent from the body of work detailed that there is already an impressive array of currently available CNT applications in neurobiology. As a result of their unique properties and the sophistication of the chemistries available for their modification, CNTs have immense potential in neuroscience. Indeed, further advances in nanotechnology will only provide greater opportunities to use these materials to expand our understanding of brain function and repair the loss of it. CNTs seem an excellent material for the construction of neuroprostheses for the repair of nerve injury, since they can be systematically modified to specifically modulate neurite outgrowth. The use of patterned arrays of CNTs to monitor neural network activity offers a possibility for bio-computing. When combined with their mechanical durability which makes CNTs well suited for use in implantable electrodes that can interact directly with the brain, it is tempting to speculate that the days of applications of CNTs in brain-machine interfaces are close. Although there have been concerns in respect to their toxicity $(28,29)$, the experimental evidence indicates that mammalian exposure to nanotubes for several weeks seems to have no adverse health effects (30). Of course, further studies are necessary before CNTs can be applied to human subjects, but at the present there is no indication that CNTs will be any more hazardous than other forms of carbon. Given their enormous potential in nanomedicine, it is imperative to continue the research on this 
unique nanomaterial using multifaceted approaches ranging from organic chemistry to translational medicine.

\section{Acknowledgments}

The authors' work is supported by a grant from the National Institute of Mental Health (MH 069791). We dedicate this paper to Glenn I. Hatton, whose energy and creativity inspired new views of astrocyte-neuronal interactions.

\section{References}

1. Radushkevich L, Lukyanovich V. O strukture ugleroda, obrazujucegosja pri termiceskom razlozenii okisi ugleroda na zeleznom kontakte. Zurn Fisic Chim 1952;26:88-95.

2. Ijima S, Ichihashi T. Single-shell carbon nanotubes of 1-nm diameter. Nature 1993;363:603-605.

3. Bethune D, Kiang C, De Vries M, Gorman G, Savoy R. Cobalt catalysed growth of carbon nanotubes with single-atomic-layer walls. Nature 1993;363:605-607.

4. Monthioux M, Kuznetsov V. Who should be given the credit for the discovery of carbon nanotubes? Carbon 2006;44(9):1621-1623.

5. Bekyarova, E.; Haddon, RC.; Parpura, V. Biofunctionalization of carbon nanotubes. In: Kumar, CSSR., editor. Biofunctionalization of nanomaterials (nanotechnologies for the life sciences). Vol. 1. Wiley; 2005.

6. Bekyarova E, Ni Y, Malarkey EB, Montana V, McWilliams JL, Haddon RC, Parpura V. Applications of carbon nanotubes in biotechnology and biomedicine. J Biomed Nanotechnol 2005;1:3-17. [PubMed: 19763242]

7. Malarkey EB, Parpura V. Applications of carbon nanotubes in neurobiology. Neurodegener Dis 2007;4:292-299. [PubMed: 17627132]

8. Mattson MP, Haddon RC, Rao AM. Molecular functionalization of carbon nanotubes and use as substrates for neuronal growth. J Mol Neurosci 2000;14:175-182. [PubMed: 10984193]

9. Hu H, Ni YC, Montana V, Haddon RC, Parpura V. Chemically functionalized carbon nanotubes as substrates for neuronal growth. Nano Lett 2004;4:507-511.

10. Hu H, Ni Y, Mandal SK, Montana V, Zhao B, Haddon RC, Parpura V. Polyethyleneimine functionalized single-walled carbon nanotubes as a substrate for neuronal growth. J Phys Chem B 2005;109:4285-4289. [PubMed: 16851493]

11. Liopo AV, Stewart MP, Hudson J, Tour JM, Pappas TC. Biocompatibility of native and functionalized single-walled carbon nanotubes for neuronal interface. J Nanosci Nanotechnol 2006;6:1365-1374. [PubMed: 16792366]

12. Matsumoto K, Sato C, Naka Y, Kitazawa A, Whitby RL, Shimizu N. Neurite outgrowths of neurons with neurotrophin-coated carbon nanotubes. J Biosci Bioeng 2007;103:216-220. [PubMed: 17434423]

13. Gheith MK, Sinani VA, Wicksted JP, Matts RL, Kotov NA. Single-walled carbon nanotube polyelectrolyte multilayers and freestanding films as a biocompatible platform for neuroprosthetic implants. Adv Mater 2005;17:2663-2670.

14. Galvan-Garcia P, Keefer EW, Yang F, Zhang M, Fang S, Zakhidov AA, Baughman RH, Romero MI. Robust cell migration and neuronal growth on pristine carbon nanotube sheets and yarns. $\mathrm{J}$ Biomater Sci Polym Ed 2007;18:1245-1261. [PubMed: 17939884]

15. Dubin RA, Callegari G, Kohn J, Neimark A. Carbon nanotube fibers are compatible with mammalian cells and neurons. IEEE Trans Nanobioscience 2008;7:11-14. [PubMed: 18334451]

16. Gabay T, Jakobs E, Ben-Jacob E, Hanein Y. Engineered self-organization of neural networks using carbon nanotube clusters. Phys a-Stat Mech Appl 2005;350:611-621.

17. Sorkin R, Gabay T, Blinder P, Baranes D, Ben-Jacob E, Hanein Y. Compact self-wiring in cultured neural networks. J Neural Eng 2006;3:95-101. [PubMed: 16705265]

18. Jan E, Kotov NA. Successful differentiation of mouse neural stem cells on layer-by-layer assembled single-walled carbon nanotube composite. Nano Lett 2007;7:1123-1128. [PubMed: 17451277] 
19. Wang K, Fishman HA, Dai H, Harris JS. Neural stimulation with a carbon nanotube microelectrode array. Nano Lett 2006;6:2043-2048. [PubMed: 16968023]

20. Lovat V, Pantarotto D, Lagostena L, Cacciari B, Grandolfo M, Righi M, Spalluto G, Prato M, Ballerini L. Carbon nanotube substrates boost neuronal electrical signaling. Nano Lett 2005;5:1107-1110. [PubMed: 15943451]

21. Mazzatenta A, Giugliano M, Campidelli S, Gambazzi L, Businaro L, Markram H, Prato M, Ballerini L. Interfacing neurons with carbon nanotubes: electrical signal transfer and synaptic stimulation in cultured brain circuits. J Neurosci 2007;27:6931-6936. [PubMed: 17596441]

22. Ali SR, Ma Y, Parajuli RR, Balogun Y, Lai WY, He H. A nonoxidative sensor based on a selfdoped polyaniline/carbon nanotube composite for sensitive and selective detection of the neurotransmitter dopamine. Anal Chem 2007;79:2583-2587. [PubMed: 17286387]

23. Zheng M, Jagota A, Semke ED, Diner BA, McLean RS, Lustig SR, Richardson RE, Tassi NG. DNA-assisted dispersion and separation of carbon nanotubes. Nature Mater 2003;2:338-342. [PubMed: 12692536]

24. Zheng M, Jagota A, Strano MS, Santos AP, Barone P, Chou SG, Diner BA, Dresselhaus MS, Mclean RS, Onoa GB, Samsonidze GG, Semke ED, Usrey ML, Walls DJ. Structure-based carbon nanotube sorting by sequence-dependent DNA assembly. Science 2003;302:1545-1548. [PubMed: 14645843]

25. Wang CW, Pan CY, Wu HC, Shih PY, Tsai CC, Liao KT, Lu LL, Hsieh WH, Chen CD, Chen YT. In situ detection of chromogranin a released from living neurons with a single-walled carbonnanotube field-effect transistor. Small 2007;3:1350-1355. [PubMed: 17576645]

26. Keefer EW, Botterman BR, Romero MI, Rossi AF, Gross GW. Carbon nanotube coating improves neuronal recordings. Nat Nanotechnol 2008;3:434-439. [PubMed: 18654569]

27. Schwartz AB, Cui XT, Weber DJ, Moran DW. Brain-controlled interfaces: movement restoration with neural prosthetics. Neuron 2006;52:205-220. [PubMed: 17015237]

28. Service RF. Nanotechnology. Sorting technique may boost nanotube research. Science 2003;300:2018. [PubMed: 12829756]

29. Service RF. Nanotoxicology. Nanotechnology grows up. Science 2004;304:1732-1734. [PubMed: 15205504]

30. Liu Z, Davis C, Cai W, He L, Chen X, Dai H. Circulation and long-term fate of functionalized, biocompatible single-walled carbon nanotubes in mice probed by Raman spectroscopy. Proc Natl Acad Sci U S A 2008;105:1410-1415. [PubMed: 18230737] 

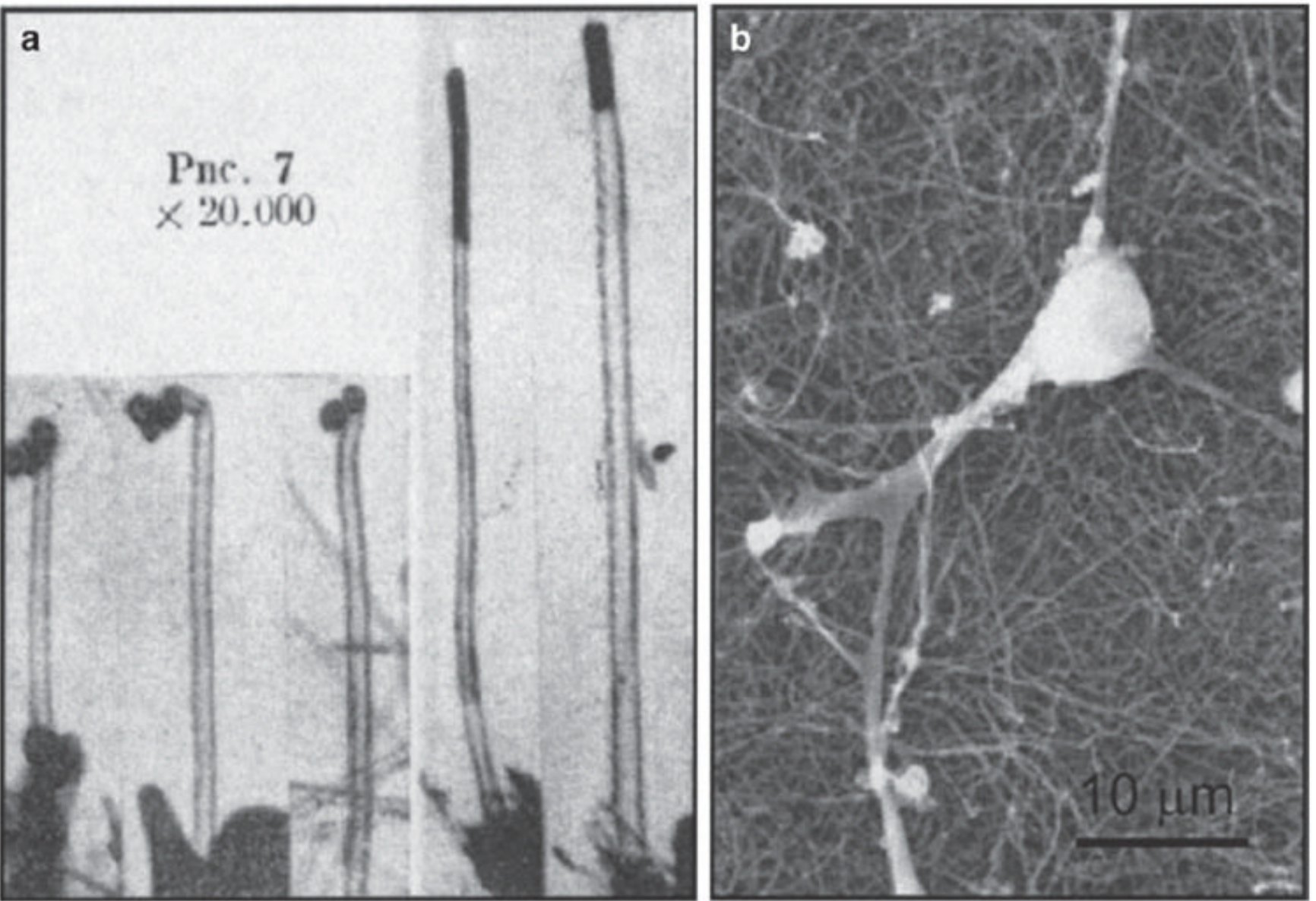

Fig. 1.

(a) Transmission electron micrograph of carbon nanotubes displaying diameters in the range of $50 \mathrm{~nm}$ (modified Fig. 7 from (1); also see commentary in (4)). (b) Scanning electron micrograph of neurons grown on "as prepared" multi-walled carbon nanotubes. Scale bar: $10 \mu \mathrm{m}$ (modified Fig. 2 from (9)) 